\title{
Use of plan curvature variations for the identification of ridges and channels on DEM
}

\author{
Sanjay Rana \\ Department of Civil and Environmental Engineering, University College London, Gower \\ Street, London WC1E 6BT, UK s.rana@ucl.ac.uk
}

\begin{abstract}
This paper proposes novel improvements in the traditional algorithms for the identification of ridge and channel (also called ravines) topographic features on raster digital elevation models (DEMs). The overall methodology consists of two main steps: (1) smoothing the DEM by applying a mean filter, and (2) detection of ridge and channel features as cells with positive and negative plan curvature respectively, along with a decline and incline in plan curvature away from the cell in direction orthogonal to the feature axis respectively. The paper demonstrates a simple approach to visualize the multi-scale structure of terrains and utilize it for semi-automated topographic feature identification. Despite its simplicity, the revised algorithm produced markedly superior outputs than a comparatively sophisticated feature extraction algorithm based on conic-section analysis of terrain.
\end{abstract}

\section{Introduction}

Ridge and channel are two fundamental features of terrain morphology. Owing to their unique significance in the shape and structure of terrains, ridge and channel features are used in various terrain analyses ranging from drainage basin delineation (see e.g., Band 1986) to intervisibility computation (see e.g., Rana 2003). In addition, as a generic abstraction of the surface structure, their use also extends in the analysis and visualization of surfaces in socioeconomic studies (see e.g., Okabe and Masuyama 2004), metrology (see e.g., Scott 2004), and computer graphics (see e.g., Belyaev and Anoshkina 2005). Naturally, an enormous amount of research has been done in the automated delineation of ridges and channels from various types of surface datasets such as triangulated meshes and raster DEMs. This paper proposes simple and novel improvements in the algorithms for ridge and channel extraction in raster DEMs, with significantly improved results.

As a background to the following discussion on various algorithms, a brief summary of the relevant aspects of feature extraction in raster DEMs is essential. In most cases of cell-based feature extraction algorithms, a terrain is studied in patches, which are square windows of $m \mathrm{x}$ $m$ cells (also called kernel or filter) of DEM, centered on the cell of interest. The value of $m$ is an odd integer greater than 2 and no more than the lesser amongst the number of rows and columns. In polynomial-fitting based algorithms, kernel is considered analogous to a set of regularly spaced points, each one typically derived from the geometric centre of the kernel cells. The result of the feature classification is assigned to a cell in the output raster, which is located at the same place as the kernel's central cell. Most feature extraction algorithms based on kernels above suffer from two fundamental limitations. As the kernel size remains fixed during a feature extraction, geographic features whose extents are not pronounced within the dimensions of the kernel (e.g., gently sloping features), could be incorrectly classified. Figure 1 shows the example of a $3 \times 3$ cells kernel on two ridges with different extents. In Figure 1a the point of maximum curvature lies within the kernel hence the central cell will be correctly classified as part of a ridge. However, in Figure $1 b$ due to its shape, the top of the ridge is not so well defined within the kernel hence although the central cell probably belongs to a large 
ridge feature it will be identified as planar. This limitation is referred as the scale-dependency of the feature extraction algorithm. Another limitation relates to the odd number of cells in the square window, such that the cells on the edges of DEM remain unclassified. For further information on raster based spatial analysis, refer to an introductory text by DeMers (2002).

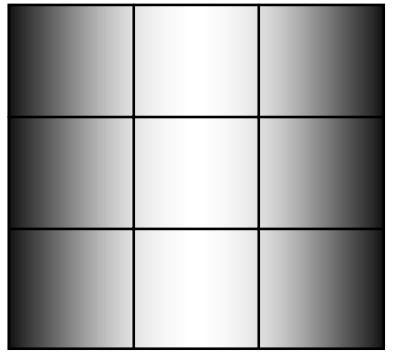

(a)

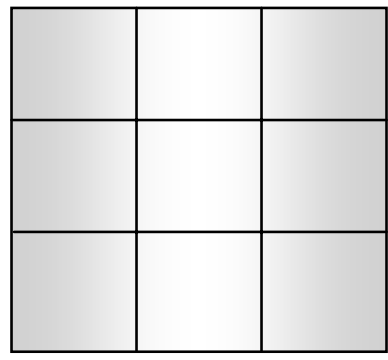

(b)

Fig. 1. Scale dependency in fixed kernel size based feature extraction. Darker color represents lower elevation and lighter color represents higher elevation. (a) Kernel can recognize the feature type as ridge and (b) Due to flattening at the ridge top, kernel recognizes it as a planar feature.

The majority of existing ridge and channel feature extraction algorithms are primarily based on either the local elevation differences or the curvature (i.e., whether convex, concave and so on), over some terrain patch. Two popular examples of local elevation differences based algorithms include the Steepest Descent or D8 (Deterministic 8) algorithm (Peucker and Douglas 1975, O'Callaghan and Mark 1984) and, the comparatively advanced algorithm based on the conic section (Wood 1996). In the D8 algorithm, a cell is considered to be a candidate channel cell if it receives flow from an adjoining cell. A cell receives flow from another cell if its elevation is lower than the other cell (i.e., upslope cell). The total flow to each cell is then accumulated by following all the flow paths and the cells that receive flow from more than a certain number of upslope cells are classified as channel cells. Ridge cells are similarly derived but without a threshold condition. In the conic section algorithm the identification of ridge and channel cells is based on the sign of the quadratic determinant of a conic section polynomial, fitted over a kernel. A nil value for quadratic determinant indicates a parabolic type of conic section, which would occur at ridge and channel areas. In the D8 algorithm, the scale i.e., the geographic area, used in the classification is typically fixed to an equivalent area of $3 \times 3$ cells kernel. Thus, features whose extent is larger than $3 \times 3$ cells area could be missed, which makes the classification scale-dependent. On the other hand, the conic section algorithm allows the sampling of elevations over variable kernel sizes, hence it can be used for multi-scale feature visualization (Wood 1996). Some other local elevation differences based algorithms include the bilinear surface patches algorithm (Schneider and Wood 2004), various D8 algorithm variants (see e.g., O’Callaghan and Mark 1984, Band 1986, Skidmore 1990). Numerous other extensions of D8 algorithms have also been proposed that enforce drainage network consistency e.g., Random 8-node (Rho8) and DEMON stream tube algorithms. For more a detailed review of these drainage network modeling related extraction algorithms, refer to Gallant and Hutchinson (2000).

Curvature based ridge and channel extraction algorithms involve a combination of first and second derivatives of elevation, namely slope and curvature respectively. Figure 2 shows a part of channel around a point $p$ and various relevant morphometric measures. Slope $(d)$ is the maximum gradient at $p$. Aspect is the direction of maximum gradient. A number of curvature measures can be derived by intersecting the terrain surface along different planes (see Wood 1996, Gallant and Hutchinson 2000 for more details on types of curvature). Maximum ( $\left.\kappa_{\max }\right)$ and minimum $\left(\kappa_{\min }\right)$ curvatures are respectively the maximum and minimum curvature along any plane. Plan curvature $\left(\kappa_{\mathrm{pl}}\right)$ at the point $p$ is the curvature of the line formed by the intersection of the terrain surface with a horizontal plane passing through $p$. Cross-section curvature $\left(\kappa_{\mathrm{cr}}\right)$ at the point $p$ is the curvature of a line formed by the intersection of a plane tangential to the terrain surface (i.e., a plane that just touches the surface) with a plane passing through $p$, bounded by the normal and strike direction (direction orthogonal to aspect) of this 
tangential plane (similar to a tilted horizontal plane). It is a general convention to convert the plan and cross-section curvature values to negative and positive values to indicate convergent and divergent flows respectively. Therefore, ridges have positive $\kappa_{\mathrm{pl}}$ and $\kappa_{\mathrm{cr}}$ values and channels have negative $\kappa_{\mathrm{pl}}$ and $\kappa_{\mathrm{cr}}$ values. Numerous variants of this fundamental rule have been published widely in Computer Graphics (see e.g., Toriwaki and Fukumura 1978, Haralick 1983, Belyaev and Anoshkina 2005, Yoshizawa et al. 2005) and GIScience literature (e.g., Smith et al. 1990, Wood 1996). For instance, a simple algorithm by Wood (1996) proposes that a point is part of ridge if $d=0 ; \kappa_{\max }>0 ; \kappa_{\min }=0$ or $d>0 ; \kappa_{\mathrm{cr}}>0$ and a point is part of channel if $d=0 ; \kappa_{\max }=0 ; \kappa_{\min }<0$ or $d>0 ; \kappa_{\mathrm{cr}}<0$. Another variant, used more commonly in computer graphics discipline (see e.g., Fisher 1989), involves the maximum ( $\left.\kappa_{1}\right)$ and minimum $\left(\kappa_{2}\right)$ curvatures (called principal curvatures) derived by intersecting all the planes that contain the surface normal with the surface. A ridge (a convex cylinder) and a channel (a concave cylinder) both have $\kappa_{2}=0$ but $\kappa_{1}>0$ and $\kappa_{1}<0$ respectively. Like the conic section based algorithm, curvature based algorithms can be used over variable kernel sizes.

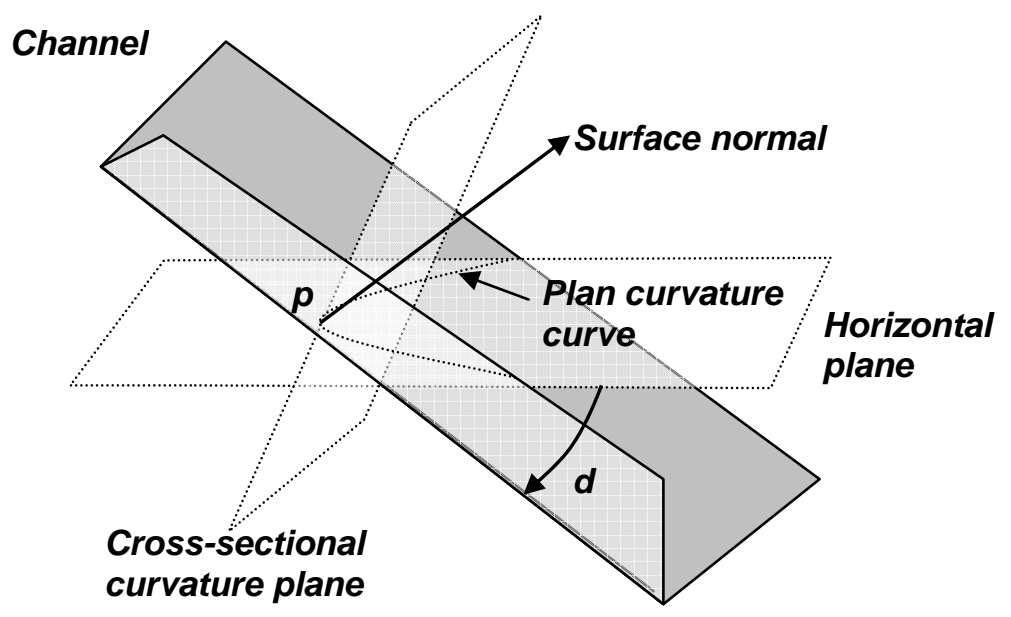

Fig. 2. A point $p$ along a channel and the various morphometric measures, namely slope (d), plan curvature curve, surface normal and the cross-sectional curvature plane.

In this we paper we propose a novel semi automated iterative algorithm, which employs the variation in plan curvature orthogonal to the ridge and channel axes as the basis for ridge and channel classification. In addition, we propose a statistical approach based on variations in the nominal counts of feature types during iterations, as a possible quantitative approach to evaluate and control the feature classification. The terms "features" and "topographic features" will be used for ridges and channels hence forth in the text.

\section{Methodology}

The overall algorithm consists of two main steps. Firstly, smoothing of the raster DEM and secondly, calculation of plan curvature and identification of topographic features. These steps are repeated until a desired feature classification has been achieved. The details on each of the steps above are given in the following sections.

\subsection{Smoothing of Raster DEM}

The removal of DEM noise is generally the first step in most feature extraction algorithms. In the case of basic steepest descent based algorithms, noise in DEM could lead to incorrect 
feature classification (see e.g., Wood 1996) and spurious pits (see e.g., Jenson and Domingue 1998). In the case of curvature based algorithms, the dependence upon the surface derivatives necessitates a smoothing of DEM prior to feature extraction to avoid noise effects. A detailed discussion on the sources and removal of DEM noise is beyond the scope of this work. Refer to Martinoni (2002) for a recent review on DEM noise. Several smoothing techniques have been proposed in the literature ranging from the simple averaging to so-called feature preserving adaptive image averaging (Belyaev and Anoshkina 2005). This work uses a simple averaging technique built in the FOCALMEAN function of ArcInfo 9.0 GIS by ESRI.

Prolonged smoothing of the DEM also affects genuine topographic features by gradual erosion of the feature edges. This aspect of smoothing is commonly employed in computer graphics and vision, to study the multi-scale structure of surfaces (see e.g. Lindeberg 1994). This is unlike the DEM cell size resampling and variable kernel size approaches used in the GIScience (Wood 1996). To our knowledge, a comparison between these different types of techniques to study the multi-scale structure of terrains remains to be established. See section 3.1.1 for a hypothesis on the multi-scale structure revealed by prolonged smoothing.

\subsection{Feature classification}

\subsubsection{Plan Curvature}

The computation of plan curvature is done within the ArcInfo 9.0. It is assumed that elevation, $z=f(x, y)$ and the plan curvature is derived by fitting the bi-variate quadratic polynomial $^{1}$ (Eq. 1) over a 3x3 cells window with cell spacing $l$, shown below (ESRI 2005):

\begin{tabular}{|l|l|l|}
\hline$Z_{1}$ & $Z_{2}$ & $Z_{3}$ \\
\hline$Z_{4}$ & $Z_{5}$ & $Z_{6}$ \\
\hline$Z_{7}$ & $Z_{8}$ & $Z_{9}$ \\
\hline
\end{tabular}

$$
\begin{aligned}
& z=a x^{2} y^{2}+b x^{2} y+c x y^{2}+d x^{2}+e y^{2}+f x y+g x+h y+i, \\
& a=\left[\left(z_{1}+z_{3}+z_{7}+z_{9}\right) / 4-\left(z_{2}+z_{4}+z_{6}+z_{8}\right) / 2+z_{5}\right] / l^{4} \\
& b=\left[\left(z_{1}+z_{3}-z_{7}-z_{9}\right) / 4-\left(z_{2}-z_{8}\right) / 2\right] / l^{3} \\
& c=\left[\left(-z_{1}+z_{3}-z_{7}+z_{9}\right) / 4+\left(z_{4}-z_{6}\right) / 2\right] / l^{3} \\
& d=\left[\left(z_{4}+z_{6}\right) / 2-z_{5}\right] / l^{2} \\
& e=\left[\left(z_{2}+z_{8}\right) / 2-z_{5}\right] / l^{2} \\
& f=\left(-z_{1}+z_{3}+z_{7}-z_{9}\right) / 4 l^{2} \\
& g=\left(-z_{4}+z_{6}\right) / 2 l \\
& h=\left(z_{2}-z_{8}\right) / 2 l \\
& i=z_{5} \\
& \kappa_{\mathrm{pl}}=-2\left(d h^{2}+e g^{2}+\text { fgh }\right) /\left(g^{2}+h^{2}\right) * 100
\end{aligned}
$$

${ }^{1}$ It is based on the formula by Zevenbergen and Thorne (1987). 


\subsubsection{Classification Rules}

The proposed algorithm extends the curvature based algorithms with additional conditions. It is proposed here that a cell belongs to a ridge if $\kappa_{\mathrm{pl}}>0 ; e=\delta \kappa_{\mathrm{pl}} / \delta t=0 ; \delta e / \delta t>0$; and a cell belongs to a channel if $\kappa_{\mathrm{pl}}>0 ; e=\delta \kappa_{\mathrm{pl}} / \delta t=0 ; \delta e / \delta t<0$, where $t$ is the direction orthogonal to feature axis. In other words, a ridge cell has a positive plan curvature and is at local maxima of plan curvature orthogonal to feature axis. In contrast, a channel cell has a negative plan curvature and is at local minima of plan curvature orthogonal to feature axis. Thus, the proposed algorithm evaluates a feature's entire extent, orthogonal to the feature axis. For simplicity in implementation and demonstration purposes, the current work assumes that ridge and channel feature axis is oriented along one of the four cardinal directions i.e., N-S, E-W, NW-SE, and NE-SW. Thus, the local maxima/minima condition merely involves a comparison between the plan curvatures of the diagonally opposite cells with the plan curvature of the cell of interest. In summary, a cell is classified as a ridge/channel cell if it has positive/negative plan curvature and highest/lowest plan curvature value amongst any pair of diagonally opposite adjacent cells. Note that this definition will also classify the peak features as ridges and pit features as channels. Since saddle features have both ridge and channel morphology, they could be classified either as ridge or channel features depending upon the last conditional statement in the software which happens to evaluate the cell. The above anomalies are actually a useful side effect as they lead to better drainage network extraction. In the present demonstration, the proposed algorithm effectively combines the techniques of D8 and curvature based algorithms.

\subsection{Experimental setup}

As mentioned earlier, the proposed algorithm has been developed in ArcInfo 9.0 using the Arc Macro Language (AML) script. A 3 x 3 cells size kernel has been used to classify DEM of three study areas, namely, Cairngorm area in Scotland (400 x 400 cells, 50 m resolution, Source: Ordnance Survey Landline, Figure 3), Salisbury Hills in SW England (502 x 501 cells, 10 m resolution, Source: Ordnance Survey Landline, Figure 4), and Round Mountain area in Nevada (451 x 485 cells, 30 m resolution, Source: USGS NED, Figure 5). As can be seen from the figures, topography of these areas varies from gently rolling hills to incised cliffs. The robustness of the algorithm was particularly tested for the following factors:

- Feature classification rule: The feature classification of the proposed algorithm is compared with the relatively sophisticated conic section based algorithm available in the FEATURE NETWORK FUNCTION of LandSerf developed by Jo Wood (URL 1). For consistency, a 3 x 3 cells kernel is used in the LandSerf with no threshold criteria e.g., curvature- and slope- tolerance and distance decay. In addition, smoothed DEMs used for the proposed algorithm is also used for the LandSerf to avoid data bias. The assessment of the feature classification quality is based on a visual comparison with the morphological structure of the original DEMs. This is done so as to determine that a) feature classification was acceptable and $b$ ) effect of smoothing on feature preservation.

- Cell size sensitivity: It has been widely established that morphometric measures are dependent upon the DEM cell size (see e.g., Chang and Tsai 1991) hence three DEMS with different cell sizes (i.e., $10 \mathrm{~m}, 30 \mathrm{~m}, 50 \mathrm{~m}$ ) are selected for the experiment.

- Relief: Due to the limited orientation and small size of the feature extraction kernel, it may suffer from scale-dependency issues. Hence the current study areas as shown in figures 3-5 are chosen for their varied relief.

All the experiments are done on an IBM ThinkPad with 512 MB RAM and Pentium M $1.2 \mathrm{GHz}$ processor. 


\section{Results}

Figures 3-5 show the feature classification derived from the proposed algorithm and LandSerf. The feature classifications based on original DEMs were significantly noisy. It was found out by trial and error that smoothing the DEMs 10 times produced the earliest most acceptable feature classification. As can be seen from figures the proposed algorithm accurately identifies most of the ridges and channels. The contrast between the outputs from the proposed algorithm and ones from LandSerf is self evident. The proposed algorithm is able to identify several features unclassified by LandSerf. In addition, the proposed algorithm is able to localise the extent (i.e., thickness) of the feature more precisely despite being only limited to 4 cardinal directions. The computation time for each of the three study areas was approximately 1 minute.

\subsection{Known issues and Future Directions}

Several interesting issues and aspirations arose during the experiments.

\subsubsection{Effect of smoothing on feature classification}

In the proposed algorithm, smoothing of the DEM is one of the key factors that affect the spatial distribution of topographic features. As can be seen in Figures 3-5, each smoothing operation changes, and generally improves, the feature classification. A simple quantitative aspatial measure of such changes in feature classification is the ratio of number of feature cells to non-feature cells (henceforth referred as feature content ratio). A plot of the changes in feature content ratio between each consecutive smoothing would reveal how the feature classification evolves with varying smoothing (Figure 6). This plot reveals when the effect of smoothing appear to introduce (or stop having) marked effects on feature classification globally. Most notably,

- At the start of the smoothing, feature classification is noisy and feature content ratio shows a steep decline. However after around 5 iterations, the change in feature content ratio in all the curves varies gradually but fluctuates increasingly with more smoothing.

- The most striking feature of the curves is that all of them behave as power series with approximately the same $\mathrm{R}^{2}$ values. This is quite intriguing and deserves further investigation as to whether this phenomenon is an artifact of the smoothing operator (since terrains themselves have dissimilar morphological structure) or whether it indirectly suggests something about the multi-scale structure of the terrain.

\subsubsection{Limitations in feature classification}

Admittedly, the present work leaves scope for future research which would improve the appearance and applicability of the feature classification. Some of these desirable improvements, which are fairly straightforward to incorporate, include:

- Several of the spurious tiny ridges and channels seen in Figures 3-4 are partly due to the 3 x 3 cells kernel size, limited feature axis choices, and absence of any slope and curvature thresholds.

- The current demonstration doesn't include any post-processing to improve the presentation of the feature classification by thinning and smoothing the shape of the ridges and channels (see e.g., Yoshizawa et al. 2005) and making the classical interlocking ridge-channel network (Werner 1988) structure of drainage networks. 


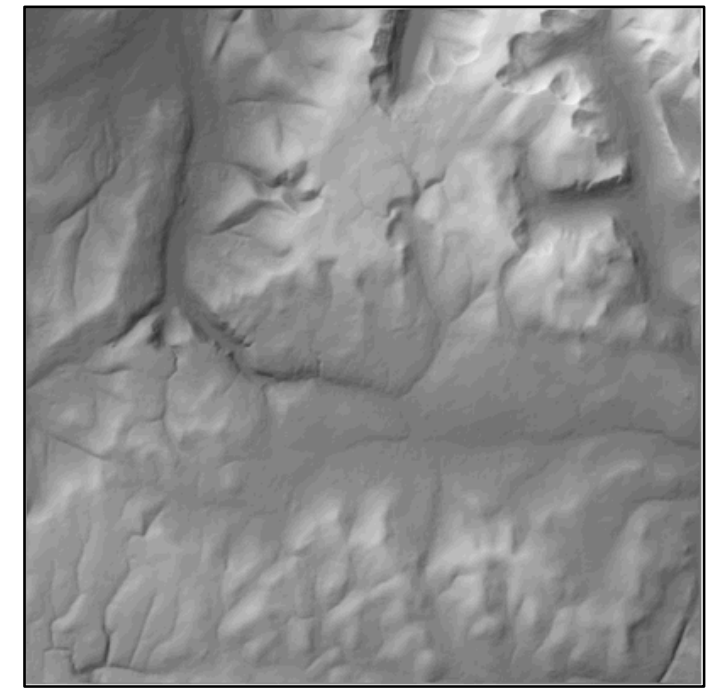

Hill shaded relief

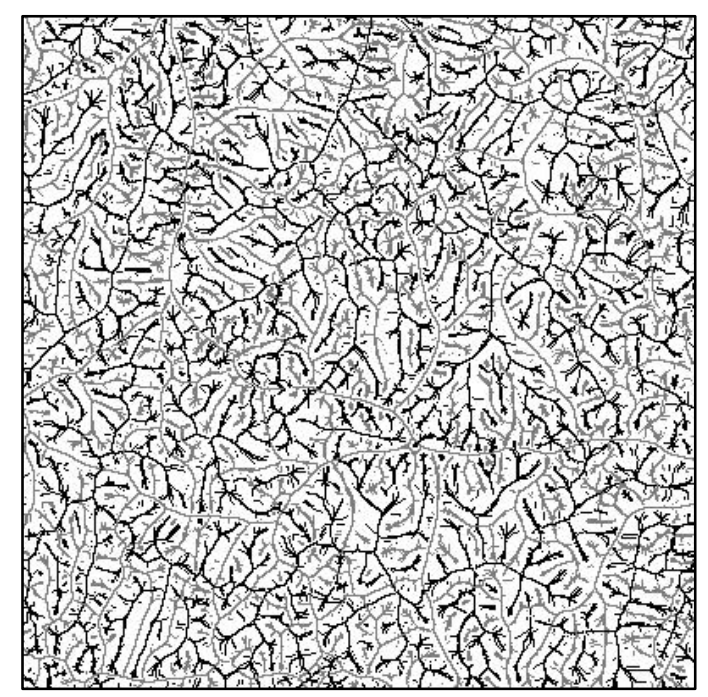

Feature classification after 10 smoothing iterations

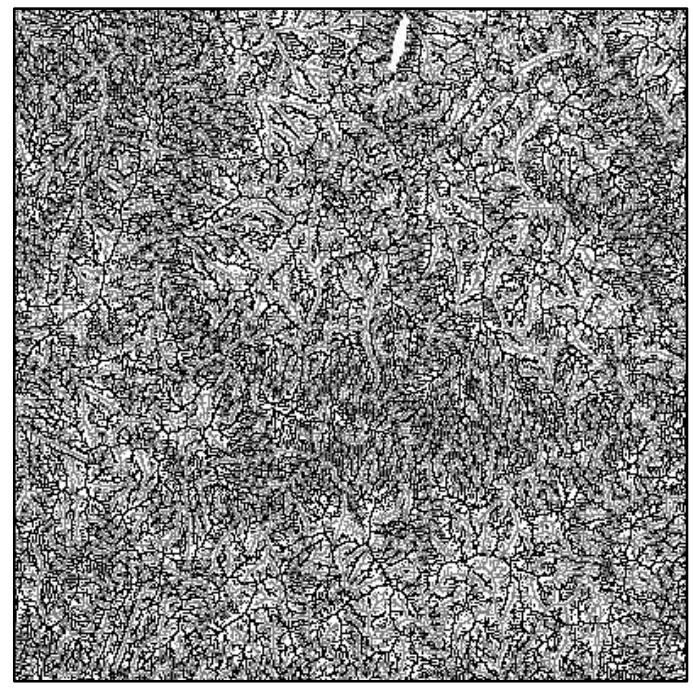

Feature classification without smoothing

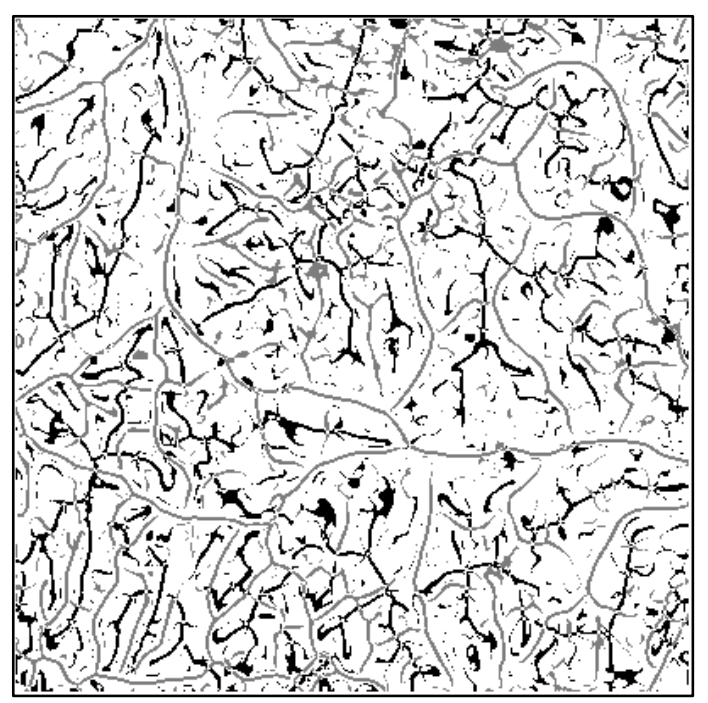

Feature classification using LandSerf, after 10 smoothing iterations

Fig. 3. Hill shaded relief of the original Cairngorm DEM and the feature classifications. Black colored cells are ridges and gray colored cells are channels. 


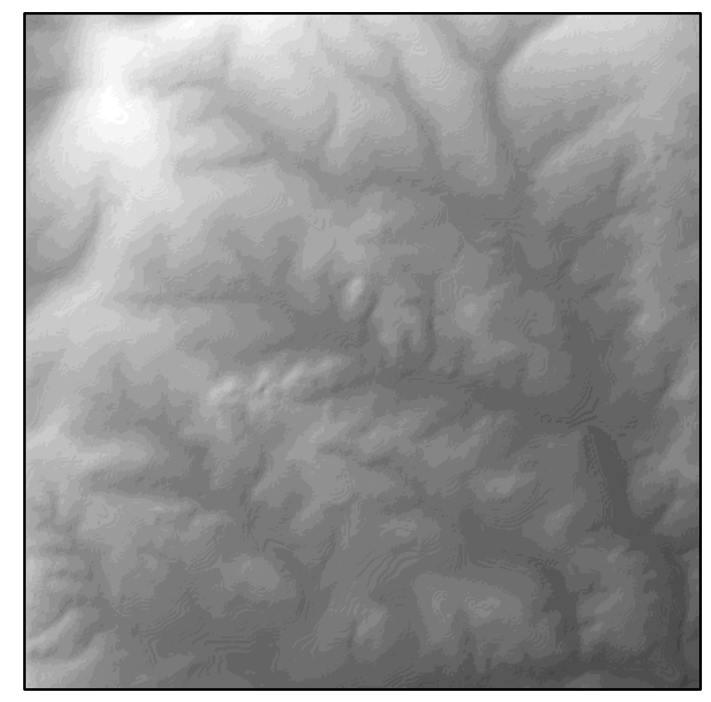

Hill shaded relief

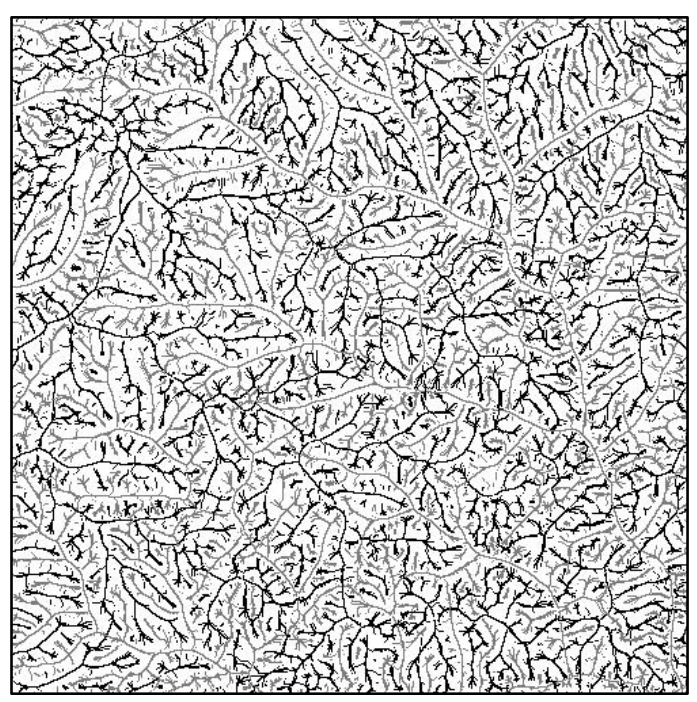

Feature classification after 10 smoothing iterations

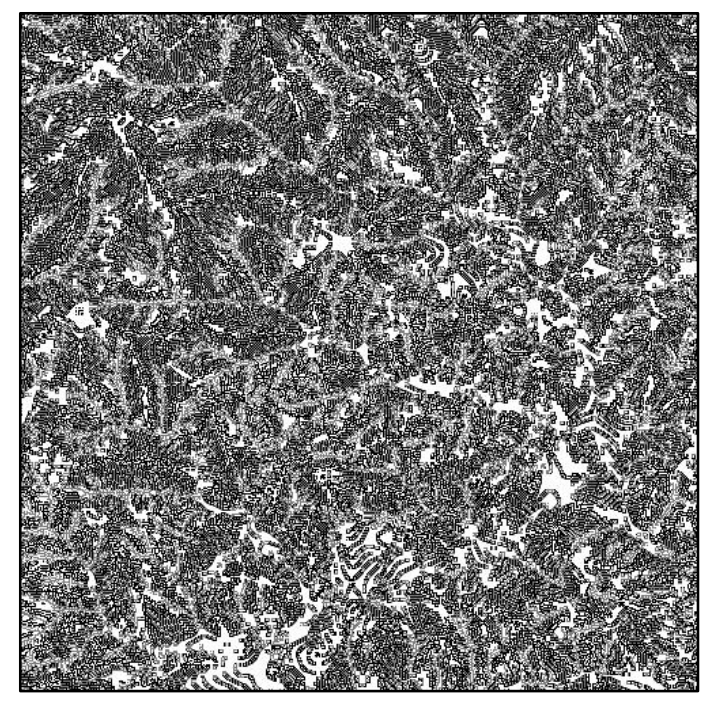

Feature classification without smoothing

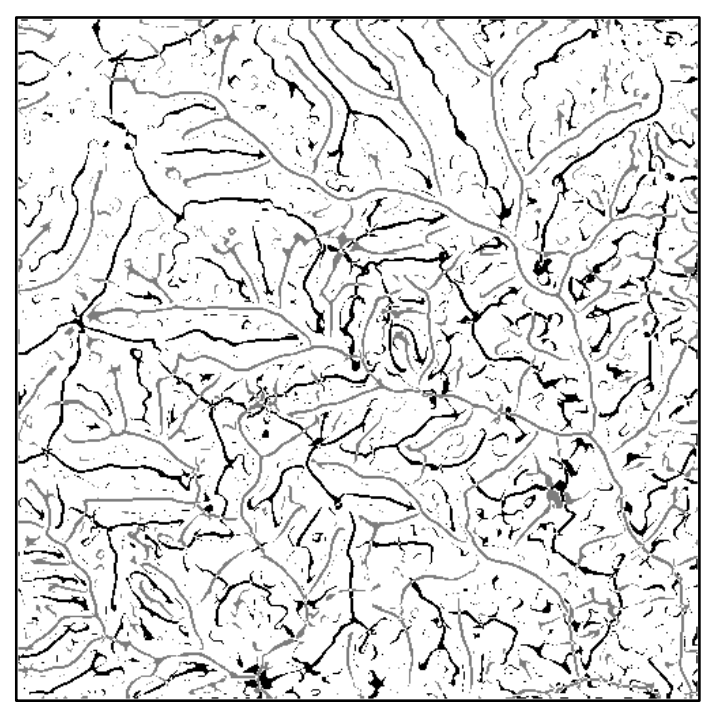

Feature classification using LandSerf, after 10 smoothing iterations

Fig. 4. Hill shaded relief of the original Salisbury DEM and the feature classifications. Black colored cells are ridges and gray colored cells are channels. 


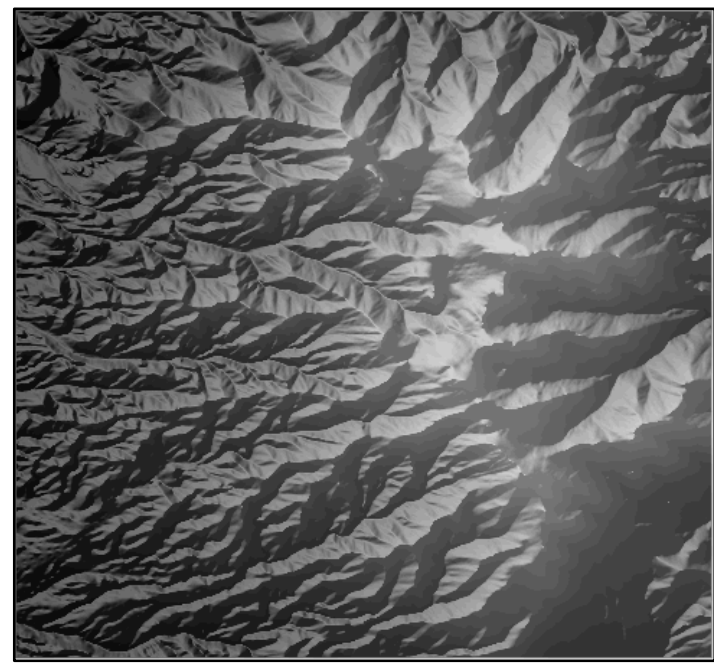

Hill shaded relief

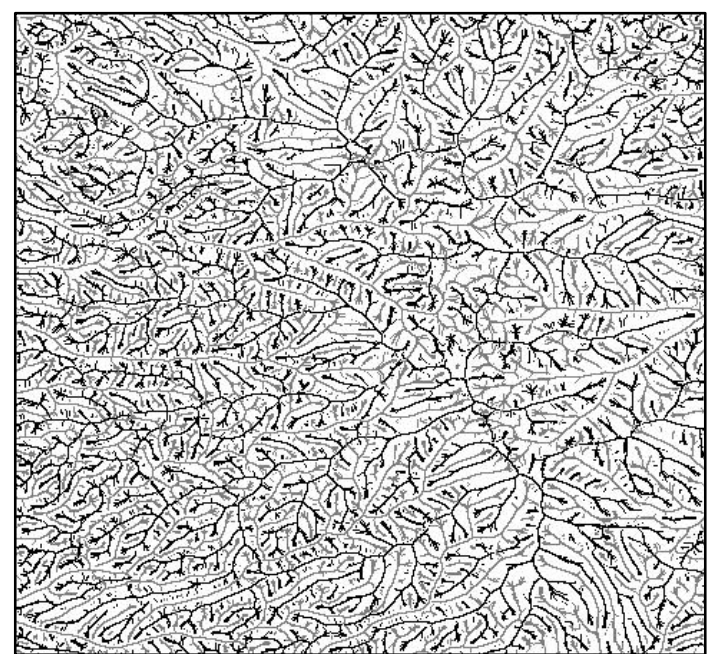

Feature classification after 10 smoothing iterations

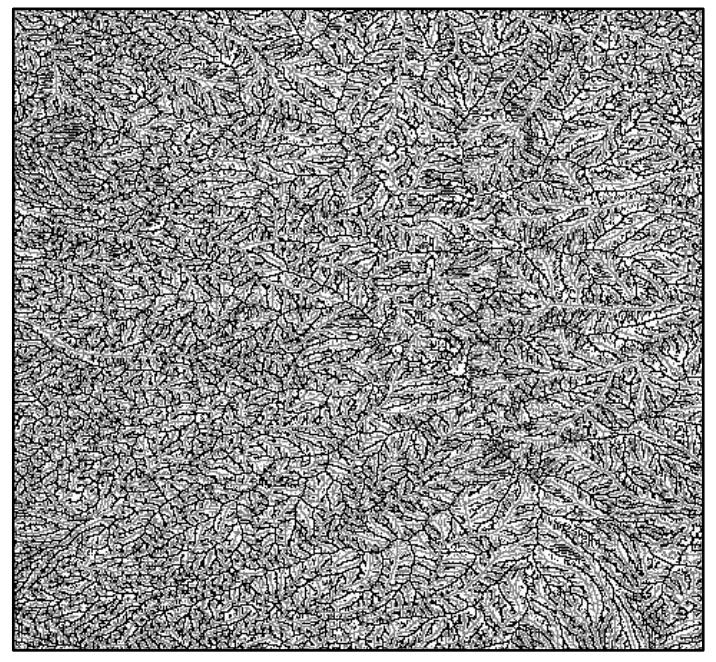

Feature classification without smoothing

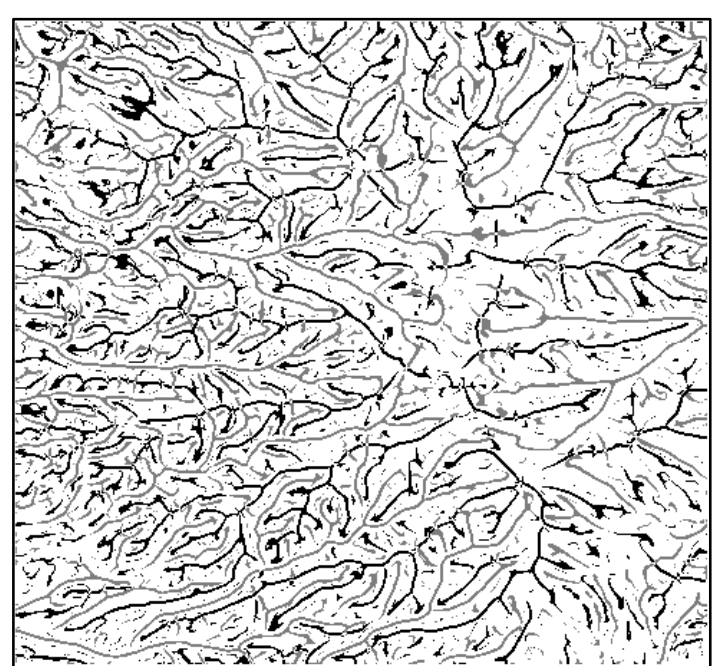

Feature classification using LandSerf, after 10 smoothing iterations

Fig. 5. Hill shaded relief of the original Round Mountain DEM and the feature classifications. Black colored cells are ridges and gray colored cells are channels. 

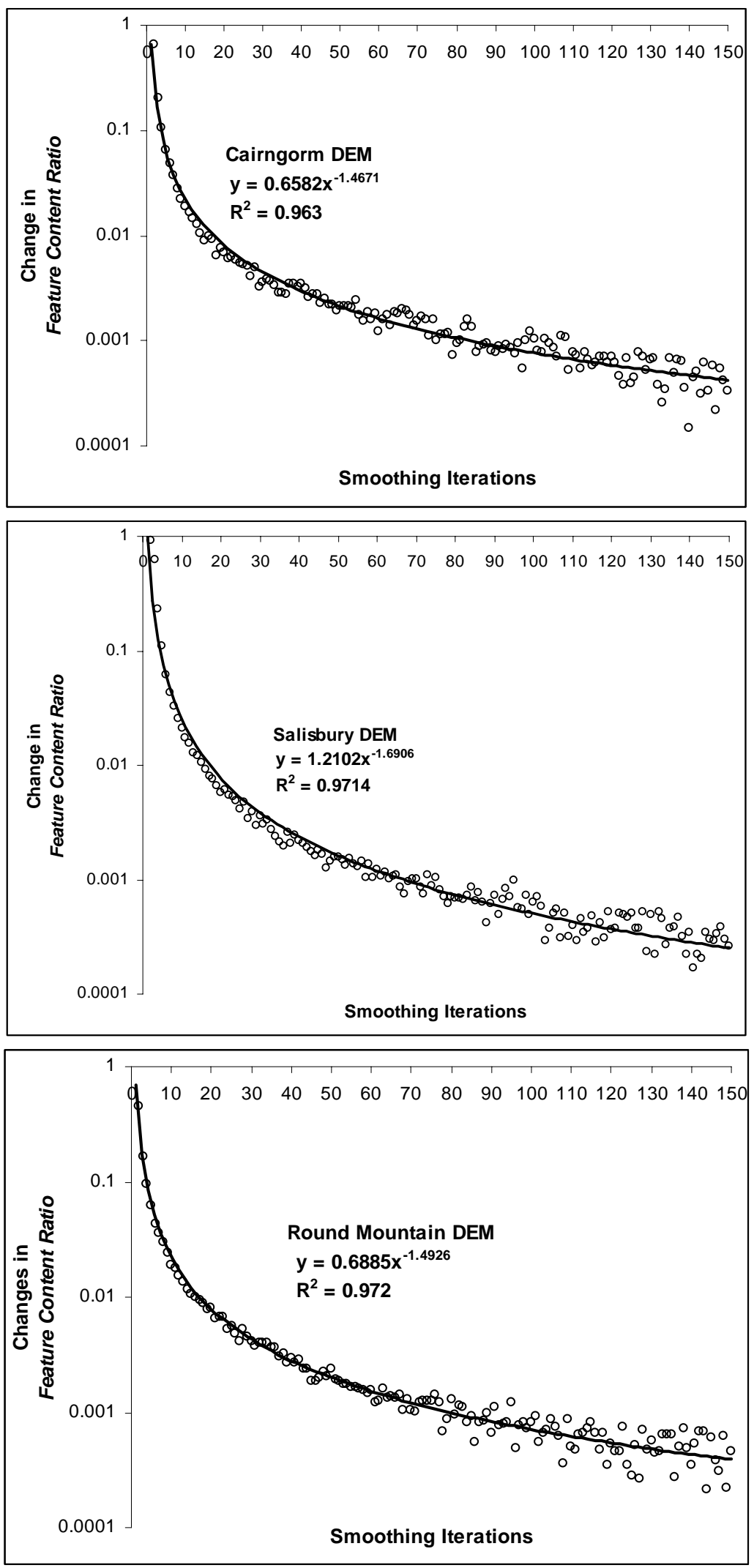

Fig. 6. Variation in the feature content ratio with smoothing iterations. 


\subsubsection{Using feature type persistence for classification}

As seen in Figures 3-5, a DEM cell could be classified differently during a sequence of smoothing operations. However, DEM cells that do not carry noise may have the same feature type for several smoothing operations. Therefore, a possible method to derive a final feature classification could be to use the most persistent feature type assigned to each cell. Wood (1996) proposed a similar approach for feature classifications derived by varying kernel sizes.

\section{Conclusions}

The paper presented an algorithm for detecting ridges and channels features in raster DEMs. It is proposed that ridge and channel features have positive and negative plan curvature respectively with descending plan curvature orthogonal to the feature axis from the higher to the lower reaches of the feature. The proposed algorithm has been demonstrated on three different areas with differing morphology and cell sizes. Despite its simplicity, the algorithm produced markedly superior outputs than a comparatively sophisticated feature extraction algorithm based on conic section modeling of terrain. The paper also evaluated the effect of smoothing on feature classification by plotting the change in the ratio of number of feature cells to non feature cells. This feature content ratio interestingly behaves as a power series.

\section{Acknowledgements}

The Ordnance Survey funded part of the research work and also provided the Salisbury DEM. Cairngorm DEM was downloaded by Young-Hoon Kim from the EDINA service. Both Salisbury and Cairngorm DEMs are Crown Copyrighted and were provided by the Ordnance Survey for purposes of research. The Round Mountain DEM was downloaded from the Seamless Data Distribution System of the USGS.

\section{References}

Band LE (1986) Topographic partition of watersheds with digital elevation models. Water Resources Research, 22:15-24.

Belyaev A, Anoshkina E (2005) Detection of surface creases in range data. In: $11^{\text {th }}$ IMA Conference on the mathematics of surfaces, Loughborough, UK.

Chang K-T, Tsai BW (1991) The effect of DEM resolution on slope and aspect mapping. Cartography and Geographic Information Systems 18:69-77.

DeMers MN (2002) GIS Modeling in Raster. John Wiley and Sons, New York.

ESRI (2005) ArcInfo GIS help on CURVATURE function.

Fisher RB (1989) From surface to objects: computer vision and three dimensional scene analysis. John Wiley and Sons, Chichester.

Gallant JC, Wilson JP (2000) Primary topographic attributes. In: Wilson JP, Gallant JC (eds) Terrain analysis: Principles and Applications, John Wiley and Sons, New York, pp 51-85.

Haralick RM (1983) Ridge and valley on digital images. Computer Vision, Graphics and Image Processing 22: 28-38.

Jenson, SK, Domingue JO (1998) Extracting topographic structure from digital elevation data for geographic information system analysis. Photogrammetric Engineering and Remote Sensing 54:1593-1600.

Lindeberg T (1994) Scale-space theory in computer vision. Kluwer Academic Publishers, Dordrecht, The Netherlands.

Martinoni D (2002) Models and experiments for quality handling in digital terrain modeling. Unpublished PhD Thesis, University of Zurich, Zurich, Switzerland.

O’Callaghan JF, Mark DM (1984) The extraction of drainage networks from digital elevation data. Computer Vision, Graphics and Image Processing 28:323-344. 
Okabe A, Masuyama M (2004) A method for measuring structural similarity among activity surfaces and its application to the analysis of urban population surfaces in Japan. In: Rana S (ed) Topological data structures for surfaces: an introduction to geographical information science, John Wiley and Sons, Chichester, pp 105-120.

Peucker TK, Douglas DD (1975) Detection of surface-specific points by local parallel processing of discrete terrain elevation data. Computers Graphics and Image Processing 4:375-387.

Rana S (2003) Fast approximation of visibility dominance using topographic features as targets and the associated uncertainty. Photogrammetric Engineering and Remote Sensing 69:881-888.

Schneider B, Wood J (2004) Construction of metric surface networks from raster-based DEMs. In: Rana S (ed) Topological data structures for surfaces: an introduction to geographical information science. John Wiley and Sons, Chichester.

Scott P (2004) An application of surface networks in surface texture. In: Rana S (ed) Topological data structures for surfaces: an introduction to geographical information science, John Wiley and Sons, Chichester, pp 157-166.

Skidmore AK (1990) Terrain position as mapped from a gridded digital elevation model. International Journal of Geographical Information Systems 4:33-49.

Smith TR, Zhan C, Gao P (1990) A knowledge-based, two step procedure for extracting channel networks from noisy DEM data. Computers \& Geosciences 16:777-786.

Toriwaki J, Fukumura T(1978) Extraction of structural information from grey pictures. Computer Graphics and Image Processing 7:30-51.

URL 1 (2005) LandSerf. http://www.landserf.org/ (accessed on 27 December 2005).

Werner C (1988) Formal analysis of ridge and channel patterns in maturely eroded terrain. Annals of the American Association of Geographers 78: 253-270.

Wood J (1996) The geomorphological characterization of digital elevation models. Unpublished PhD Thesis, University of Leicester, UK.

Yoshizawa S, Belyaev A, Seide H-P (2005) Fast and robust detection of crest lines on meshes. In: ACM Symposium on solid and physical modeling, Cambridge, USA.

Zevenbergen LW, Thorne CR (1987) Quantitative analysis of land surface topography. Earth Surface Processes and Landforms 12:47-56. 